\title{
Rheology and Shear-Induced Textures of Silver Nanowire Lyotropic Liquid Crystals
}

\author{
Teng $X u$ and Virginia A. Davis \\ Department of Chemical Engineering, Auburn University, Auburn, AL 36849, USA \\ Correspondence should be addressed to Virginia A. Davis; davisva@auburn.edu
}

Received 6 September 2015; Accepted 26 November 2015

Academic Editor: Luca Valentini

Copyright ( $) 2015$ T. Xu and V. A. Davis. This is an open access article distributed under the Creative Commons Attribution License, which permits unrestricted use, distribution, and reproduction in any medium, provided the original work is properly cited.

\begin{abstract}
We report the rheological and microstructural shear response of a mixture of polyvinylpyrrolidone (PVP) coated silver nanowires and nanoparticle aggregates (Ag) dispersed in ethylene glycol (EG) or water $\left(\mathrm{H}_{2} \mathrm{O}\right)$. Biphasic and liquid crystalline dispersions exhibited rheological characteristics similar to those of lyotropic liquid crystalline polymers (LCPs). The viscosity versus concentration curve for Ag-EG dispersions showed a viscosity minimum at a silver concentration between 2.2 vol.\% and 2.9 vol.\%; this is indicative of the transition to an entirely liquid crystalline phase. The rheology results were consistent with optical microscopy observations that at $2.9 \mathrm{vol} . \%$ the sample was entirely birefringent; this is another indication of liquid crystalline phase formation. Shear had a significant effect on the microstructure of the dispersions and dried coatings. Depending on the shear rate, worm or shear banding Structures were observed.
\end{abstract}

\section{Introduction}

Inorganic nanocylinders (i.e., nanorods, nanowires, and nanotubes) are promising materials due to their excellent plasmonic, optoelectronic, and catalytic properties [1-3]. There have been ongoing improvements in the reproducibility and scalability of nanocylinder synthesis, but scalable methods for controlled assembly over large areas remain a persistent challenge. Fluid phase processing methods are a promising route; both the scalability and infrastructure for producing coatings from complex fluid dispersions are well established [4-8]. In addition, many nanocylinders are synthesized in a liquid dispersion. Experimental results have shown that nanocylinder coatings and films with controlled, uniform orientation have significantly improved optical [ 9 , 10] and electrical [11-13] properties, and they can be used as building blocks for functional devices. However, controlling orientation in nanocylinder coatings requires understanding dispersions' rheological and microstructural response to shear.

Silver nanowires' excellent optical, electrical, thermal, and antimicrobial properties have caused them to be explored for transparent conductive displays, solar cell components, surface-enhanced Raman scattering (SERS) substrates, electrochemical capacitors, and antimicrobial surfaces [14-17]. In our previous work, we reported demixed nematic liquid crystalline phase formation in a system comprised of polyvinylpyrrolidone (PVP) coated silver nanowires and nanoparticle aggregates dispersed in ethylene glycol/water $\left(\mathrm{EG} / \mathrm{H}_{2} \mathrm{O}\right)$ [18]. In this work, we report the results of more detailed studies on the rheological and microstructural response of these dispersions to shear. The rheological properties are analogous to those reported for classical rod-like liquid crystalline polymers (LCPs) [19-22]. We also report that shearing the dispersions results in complex microstructures. For example, applying a shear rate of approximately $200 \mathrm{~s}^{-1}$ results in a worm-like texture in biphasic dispersions; this texture is stable for several hours due to the long relaxation time of the high aspect ratio Ag nanocylinders. At higher shear rates, the silver nanowires align at the top of the coating while the spherical aggregates pack at the bottom of the coating and shear banding can occur. This intriguing range of microstructures is promising for the controlled assembly of not only silver dispersions, but also multicomponent dispersions of nanocylinders and nanoparticles. 


\section{Experimental Section}

2.1. Synthesis. Silver nanowires were synthesized using the microwave-assisted polyol method [23]. In a typical experiment, $90 \mathrm{mg}$ of silver nitrate $\left(\mathrm{AgNO}_{3}\right), 110 \mathrm{mg}$ of polyvinylpyrrolidone (PVP) (MW 58,000), and $5 \mathrm{mg}$ of sodium chloride $(\mathrm{NaCl})$ were dissolved in $20 \mathrm{~mL}$ of ethylene glycol (EG) in a $100 \mathrm{~mL}$ beaker; all chemicals were purchased from Sigma-Aldrich (Milwaukee, WI) and used as received. The solution was bath sonicated for 5 minutes in a $55 \mathrm{kHz}$ ColeParmer bath sonicator to speed the dissolution process. After all chemicals were dissolved, the solution was heated for 3.5 minutes at a power level of 3 in a household General Electric $1000 \mathrm{~W}, 2.45 \mathrm{GHz}$ microwave. It should be noted that the dispersions heat very rapidly and care needs to be exercised to avoid boiling over the dispersion or creating a significant quantity of potentially ignitable vapor. After heating, the dispersions were left in the microwave to cool for 10 minutes to avoid disturbing the growth process and reduce the risk of exposure to the hot EG. As described in Murali et al. (2010), the synthesis results in a mixture of silver nanowires and large nanoparticle aggregates [18]. To partially fractionate the dispersions and remove excess PVP and other chemicals, the dispersions were concentrated by sedimentation for at least 48 hours and then the supernatants were removed. The sediment was then washed with acetone and then with deionized water with a 20 -minute $2000 \times \mathrm{g}$ centrifugation between each washing step. The final sediment was transferred to $\mathrm{EG} / \mathrm{H}_{2} \mathrm{O}$ by repeating the centrifugation process twice in $\mathrm{EG} / \mathrm{H}_{2} \mathrm{O}$ at $2000 \times \mathrm{g}$ for 20 minutes.

2.2. Characterization. To determine the sample concentration, thermogravimetric analysis (TGA) was performed in a TA Instruments Q500 thermal gravimetric analyzer. The sample was heated in a clean platinum pan at $5^{\circ} \mathrm{C} / \mathrm{min}$ to $500^{\circ} \mathrm{C}$ under a constant Argon balance protection flow rate of $10 \mathrm{~cm}^{3} / \mathrm{min}$ and sample argon flow rate of $90 \mathrm{~cm}^{3} / \mathrm{min}$. After the temperature reached $500^{\circ} \mathrm{C}$, the temperature was held constant for 20 minutes.

Rheological characterization was performed using an Anton Paar MCR 301 rotational rheometer equipped with a P-PTD200 Peltier temperature control device and H-PTD200 Peltier upper oven at $20^{\circ} \mathrm{C}$. To increase the accessible shear rate range, a $50 \mathrm{~mm}$ parallel plate fixture was used for low concentration dispersions and a $25 \mathrm{~mm}$ parallel plate fixture was used for high concentration dispersions. For steady shear measurements, a presehear at a constant shear rate of $1 \mathrm{~s}^{-1}$ for 1000 seconds was applied to each sample after loading to ensure a well defined shear history. The temperature of the samples was controlled at $20^{\circ} \mathrm{C}$ for all the rheology tests.

Dispersion microstructure was characterized using a Nikon Eclipse 80i color transmission optical microscope equipped with cross-polarized differential interference contrast (DIC), a Retiga camera, and a digital imaging workstation with NIS-Elements software. Coating morphology was characterized by both a JEOL 7000-F field emission scanning electron microscope (SEM) and optical microscopy using reflected cross-polarized light. Coating electrical properties were characterized by a Keithley 4200-SCS semiconductor characterization system with the four-point probe method.

\section{Results and Discussion}

3.1. Synthesis. After the sedimentation and washing process, the size distributions of the samples used in this research were characterized using optical microscopy and SEM (Figure 1). Size measurements made on more than 5000 entities using optical microscopy showed significant nanowire length polydispersity with an average length $\langle L\rangle=4.8 \mu \mathrm{m}$; the number fraction of silver nanowires was approximately 0.05. SEM characterization of the nanowire and nanoparticle aggregate diameters showed a broad diameter distribution of silver nanoparticle aggregates with $\langle D\rangle=170 \mathrm{~nm}$ and relatively narrow diameter distribution of silver nanowires with $\langle D\rangle=$ $60 \mathrm{~nm}$. This results in a silver nanowire average aspect ratio $\langle L / D\rangle=80$.

3.2. Dispersion Morphology. The dispersion microstructure of silver nanowire and nanoparticle aggregates in ethylene glycol at different concentrations was studied by a crosspolarized optical microscopy (Figure 2). Based on crosspolarized optical microscopy, the isotropic-biphasic transition $\phi_{I}$ was 0.1 vol. $\%$ (total silver nanomaterials). As described in our previous work [18], the microstructure of silver nanowires in this biphasic region showed a strand-like morphology which has been previously observed in biphasic dispersions of single-walled carbon nanotubes (SWNT) in superacids [24]. In accordance with liquid crystalline phase behavior, as the silver concentration increased, the fraction of birefringent liquid crystalline domains increased. It is interesting to note that there was a dramatic microstructure change at silver volume fraction between 0.6 and 0.8 vol. \%. The microstructure of Ag-EG with 0.8 vol.\% silver showed more significant networking than that of Ag-EG with 0.6 vol. $\%$ silver. When the silver volume fraction reached approximately $2.8 \mathrm{vol} . \%$, a single-phase polydomain lyotropic liquid crystal formed in EG. This entirely liquid crystalline phase is a demixed nematic containing both strongly birefringent rod-rich regions and sphere-rich (dark brown) regions. Rod-sphere demixing was also reported in our previous work [18] as well as modeling results for dispersions of polymer spheres and rod-shape $f d$ virus [25].

3.3. Rheology. Rheology is a useful tool to probe the microstructure and shear response of liquid crystalline phases of rod-like polymers [19-22] and nanocylinders [18, 24, 26-31]. Rheological features for rod-like polymer lyotropic liquid crystals include the following: the viscosity versus concentration curve has a maximum; the response of startup shearing tests shows that the viscosity and first normal force difference oscillate over 100 shear units (time multiplied by shear rate) before reaching the steady state; the viscosity versus shear rate curve often shows three distinct regions; and the Cox-Merz rule is not obeyed.

For isotropic Ag-EG dispersions, the start-up of constant shear showed an overshoot followed by steady state in less than 100 shear units as reported for other isotropic systems 


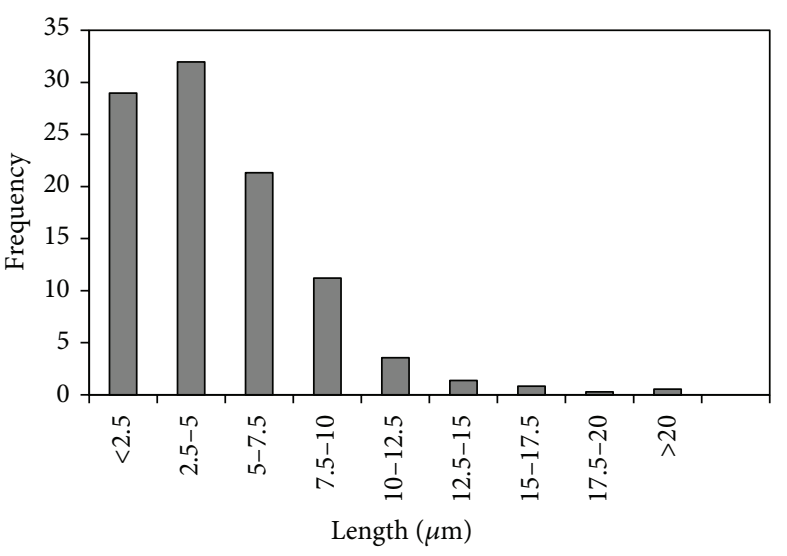

(a)

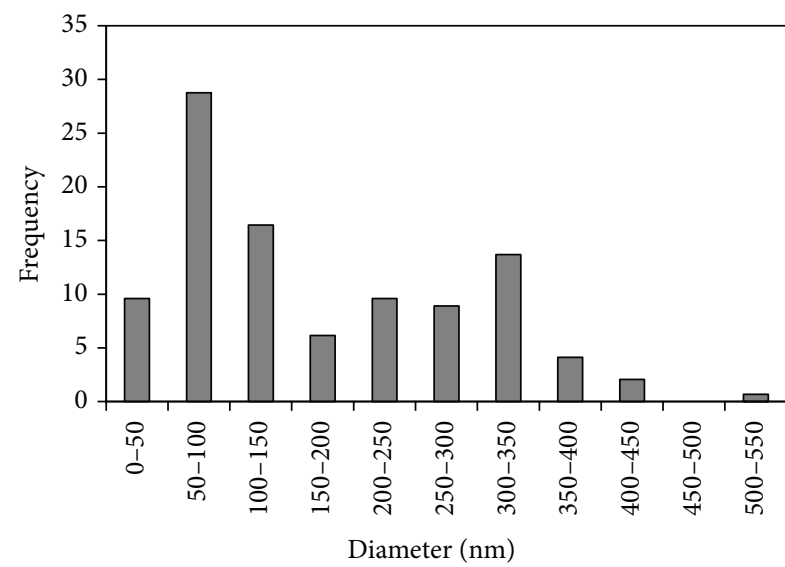

(c)

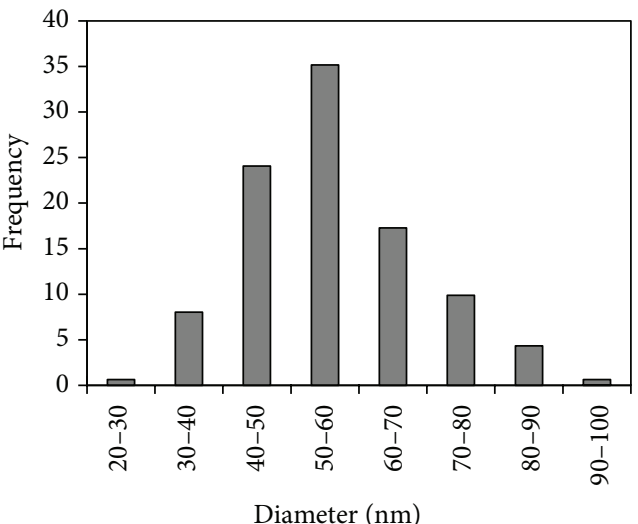

(b)

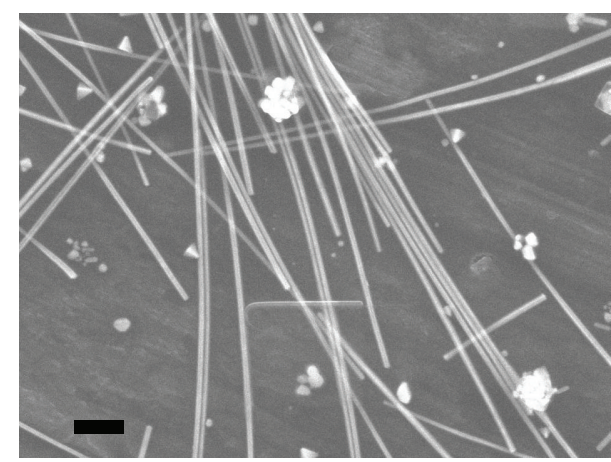

(d)

FIGURE 1: Histograms of silver nanowire (a) length and (b) diameter distributions and (c) silver nanoparticle diameter distribution. (d) SEM image of silver nanowires and nanoparticle aggregates system. The scale bar in the SEM image is $500 \mathrm{~nm}$.

[32]. However, for biphasic and liquid crystalline Ag-EG dispersions with silver volume fraction $\phi>1.90$ vol.\%, the start-up of shear resulted in an overshoot followed by uniform oscillatory transients for over 1000 shear units. The first normal stress difference N1 showed similar oscillations but also some drift. For example, Figure 3 shows the response of the start-up shear for $1.90 \mathrm{vol} \% \mathrm{Ag}$-EG at a constant shear rate of $1 \mathrm{~s}^{-1}$. The response of both viscosity and first normal stress difference showed very uniform long oscillatory transients which persisted for over 1500 shear units. Similar behavior has also been reported with other lyotropic nanocylinder systems including SWNT-102\% $\mathrm{H}_{2} \mathrm{SO}_{4}$ [24], $\mathrm{Ag}-\mathrm{H}_{2} \mathrm{O}$ [18], and silica nanorods in DMSO [31].

Figure 4 highlights the significant effect high aspect ratio rods have on low shear viscosity. It shows that, at low shear, there was six-order-of-magnitude range of viscosities for AgEG concentrations ranging from 0.6 to 4.1 vol.\%; the low viscosity of the $0.6 \mathrm{vol} . \%$ dispersion limited the accessible shear rate range to $>0.1 \mathrm{~s}^{-1}$. At an $\mathrm{Ag}$ concentration of $0.6 \mathrm{vol} . \%$, the dispersion was isotropic and behaved as a Newtonian fluid. Increasing concentration to $0.8 \mathrm{vol}$.\% resulted in both a threeorder-of-magnitude increase in low shear viscosity and shear thinning behavior. This change in rheological behavior was consistent with the optical microscopy observations that a network structure formed at 0.8 vol.\%. At this concentration and above, all of the dispersions exhibited at least one region of power law behavior $\eta=K \dot{\gamma}^{n-1}$, where $\eta$ is viscosity, $\dot{\gamma}$ is the shear rate, $K$ is the consistency index and $n$ is the power law index. Consistent with observations for other macromolecular liquid crystals, several of the dispersions contained distinct low shear and high shear power law regions with an inflection point or plateau between them. This three-region behavior is associated with the polydomain texture of nematic liquid crystals [33]. The low shear Region I is associated with director tumbling, the intermediate shear Region II is associated with director wagging or vorticity alignment, and the highest shear Region III is associated with flow alignment. The low shear viscosity kept increasing with Ag concentration until 1.8 vol.\%; it then decreased until a concentration of $2.2 \mathrm{vol} . \%$ and then increased again. This nonmonotonic behavior is another feature commonly observed for macromolecular lyotropic liquid crystals including LCPs, SWNT- $\mathrm{H}_{2} \mathrm{SO}_{4}$ [24], dsDNA/SWNT- $\mathrm{H}_{2} \mathrm{O}$ [26], and $\mathrm{SiO}_{2}-$ DMSO [31] dispersions. For an isotropic dispersion, viscosity increases with concentration. However, in a lyotropic dispersion, once the fraction of aligned liquid crystal domains 


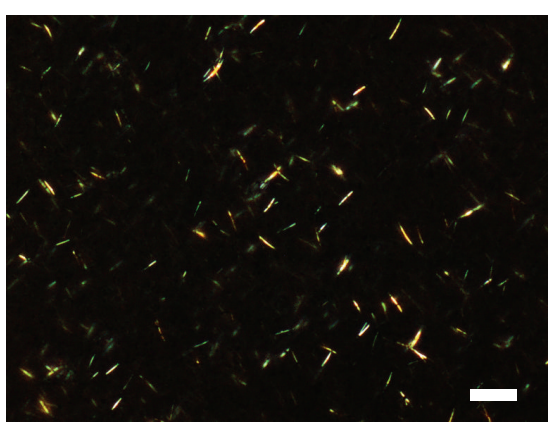

(a)

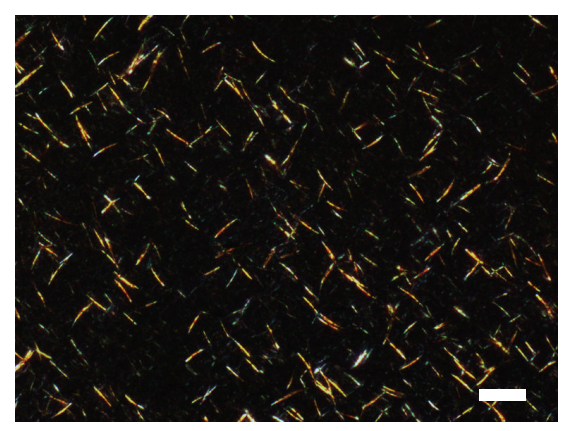

(b)

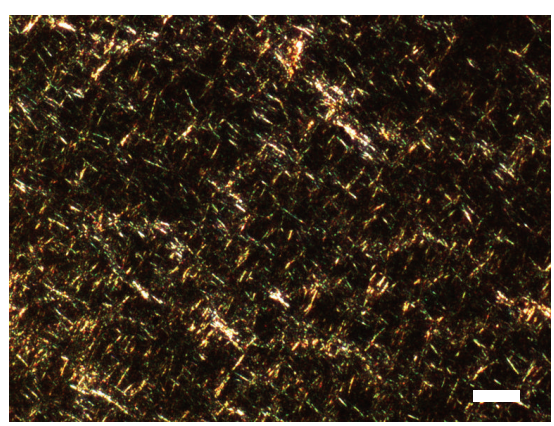

(c)

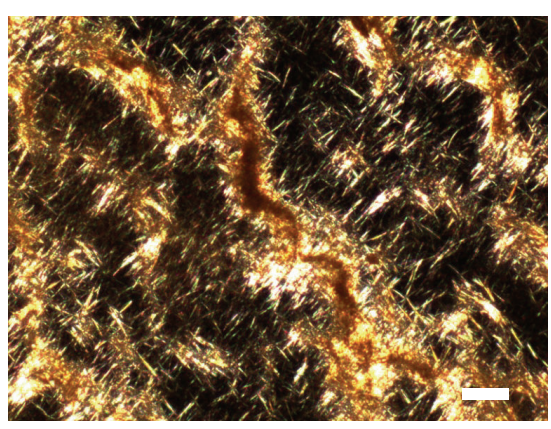

(d)

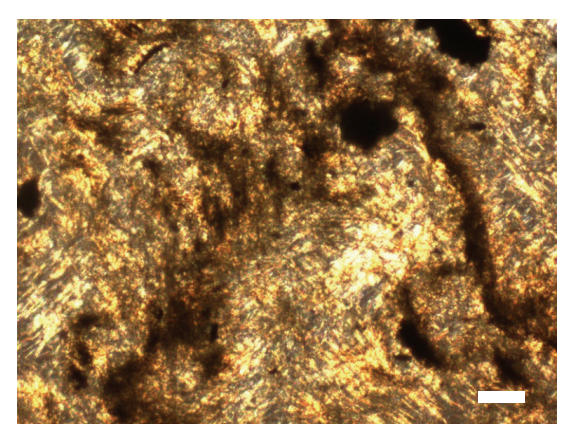

(e)

FIGURE 2: Color transmission microscopy images of Ag-EG dispersions at different concentration under cross-polarized light using DIC and a 20x $0.45 \mathrm{NA}$ objective. The total silver nanowire and nanoparticle aggregates concentrations were (a) $0.1 \mathrm{vol} . \%$, (b) $0.6 \mathrm{vol} . \%$, (c) 0.8 vol.\%, (d) 2.2 vol. $\%$, and (e) 2.8 vol. \%. Scale bar value is $20 \mu \mathrm{m}$.

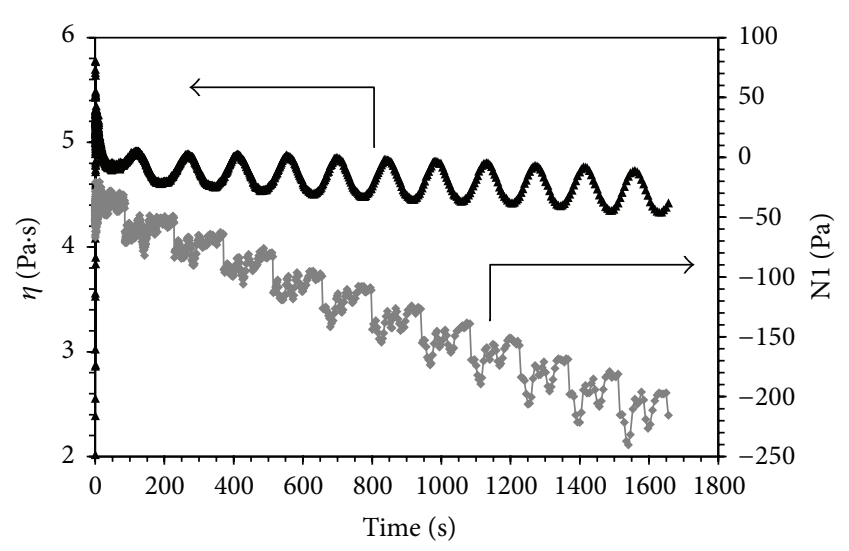

FIGURE 3: The response of the start-up of shear at $1 \mathrm{~s}^{-1}$ for a 1.9 vol.\% Ag-EG dispersion shows oscillatory transients for both viscosity (black data) and the first normal force difference (gray data) with time.

is sufficient to dominate behavior, the viscosity decreases with concentration. The increasing fraction of aligned liquid crystalline domains results in a lower resistance to flow. Once a liquid crystal phase is fully formed, further increases in concentration result in a rapid increase in viscosity due to the increasingly tight packing of the rods. This behavior is highlighted by the viscosity versus concentration curves (Figure 5(a)) obtained at $0.1,1$, and $10 \mathrm{~s}^{-1}$. The viscosities go through a maximum at every shear rate; as expected the magnitudes of the maxima decrease with increasing shear rate. As was previously observed for lyotropic PBLG solutions, the viscosity drops abruptly after the maximum and then increases again [34]. The transition from a biphasic system to a single phase liquid crystal occurs at a concentration near the resulting upper minimum in viscosity [35]. Thus, for the Ag-EG dispersion, the biphasic to single-phase liquid crystalline transition $\phi_{N}$ occurred between 2.2 vol.\% and $2.9 \mathrm{vol} . \%$; this is consistent with the optical microscopy result shown in Figure 2. This $\phi_{N}$ transition is much lower than the Onsager theory prediction of $\phi_{N} \sim 4.5 \mathrm{vol} . \%$ for monodisperse spherocylinders interacting only through hard rod repulsion. The discrepancy is attributed to demixing, attractive forces, and polydispersity $[18,36]$.

Interestingly, when the viscosities were close to the maximum in the biphasic region (1.7 and 1.8 vol.\%) and high concentration liquid crystalline region (3.7 and 4.1 vol.\%), the flow curve showed only one shear thinning region with no inflection point. The data suggested that the dispersions were strongly yielding, which is consistent with previous observations for LCPs. Figure 5(b) highlights the evolution of yield stress with concentration. Yield stress is the static stress required to deform the director field to one in equilibrium with the imposed flow; systems that undergo more than an order of magnitude decrease in viscosity at a nearly constant stress are considered to have a yield stress. All of the dispersions containing concentrations greater than or equal to 1.2 vol. $\%$ Ag exhibited a yield stress. However, as was the case for viscosity, the magnitude of the yield stress did 


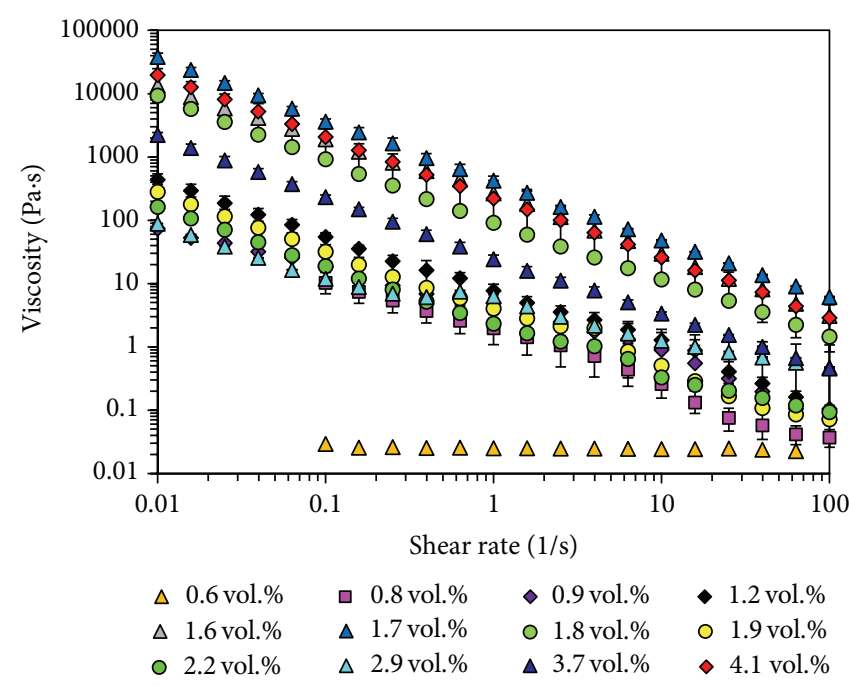

(a)

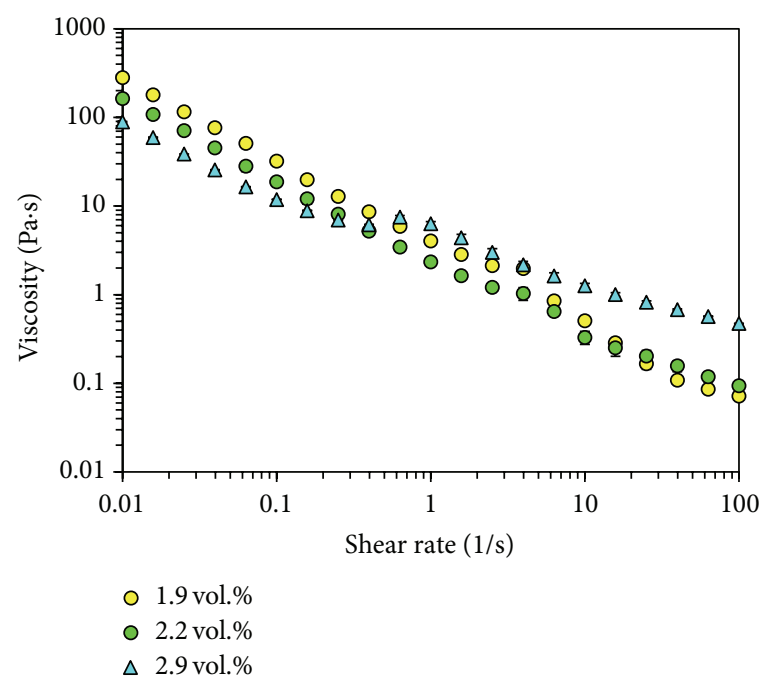

(b)

FIGURE 4: (a) The steady shear viscosity versus concentration curve of Ag-EG dispersions at different Ag concentrations. (b) Viscosity versus concentration curve of Ag-EG dispersions near the viscosity minimum.

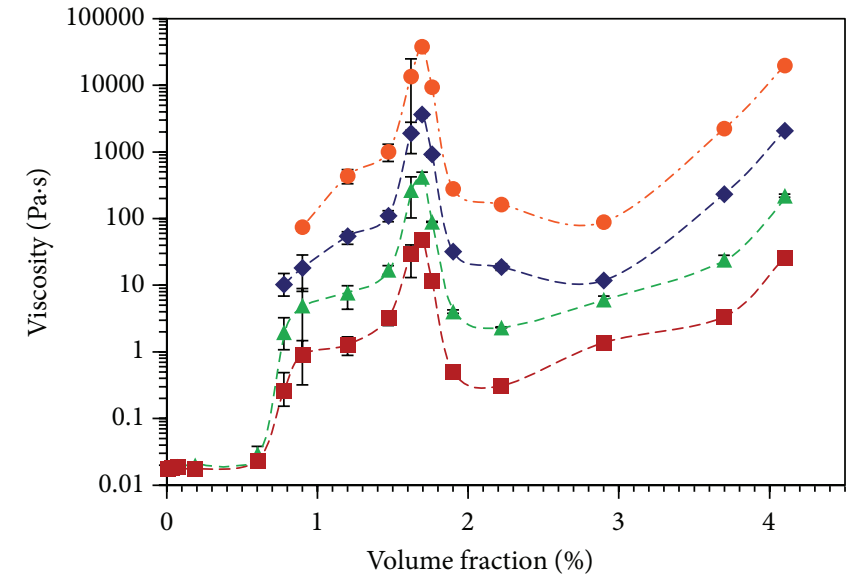

(a)

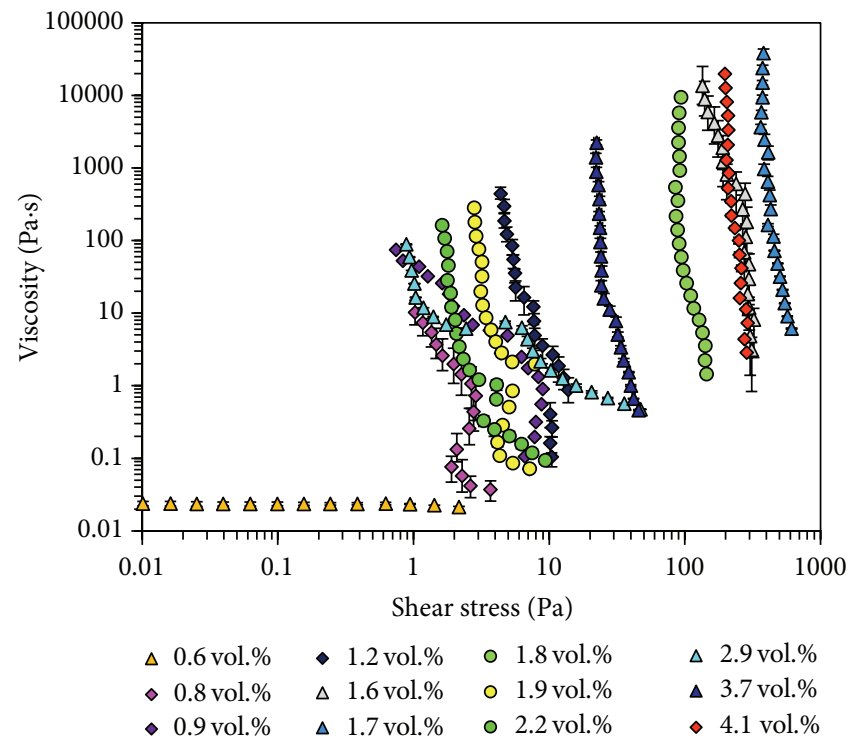

(b)

FIGURE 5: (a) Relationship between viscosity and total Ag volume fraction in EG at a shear rate of 0.01 (orange circles), 0.1 (blue diamonds), 1 (green triangles), and 10 (red squares) $\mathrm{s}^{-1}$. (b) Viscosity versus shear stress curves for Ag-EG dispersions showed a yield stress at concentrations greater than 0.8 vol. $\%$.

not vary monotonically with concentration; the yield stress reached a maximum at 1.7 vol. $\%$ and a minimum at 2.9 vol. $\%$.

Another behavior associated with LCPs is that the empirical Cox-Merz rule is not obeyed. According to the CoxMerz rule, at the same shear rate and angular frequency, the steady shear viscosity and complex viscosity should overlap. This rule is not obeyed for lyotropic liquid crystalline polymers in the anisotropic regime [21,37]. Figure 6 shows an example of the failure of the Cox-Merz rule for a 1.6 vol.\%. Ag-EG dispersion. Interestingly, the two curves showed bigger deviation at lower shear rates than that at higher shear rate. The failure of the Cox-Merz rule is additional evidence of liquid crystalline phase formation in the silver nanowire/nanoparticle aggregate dispersions.

3.4. Dependence of Texture on Shear Rate. The rheological behavior of the dispersions is indicative of changes in microstructure with shear rate. These changes were visualized using optical and scanning electron microscopy; to facilitate solvent removal analogous dispersions of $\mathrm{Ag}$ in $\mathrm{H}_{2} \mathrm{O}$ were used. For example, an Ag- $\mathrm{H}_{2} \mathrm{O}$ biphasic dispersion with 1.1 vol. $\%$ Ag was sheared at shear rate of $200 \mathrm{~s}^{-1}$ and then dried 


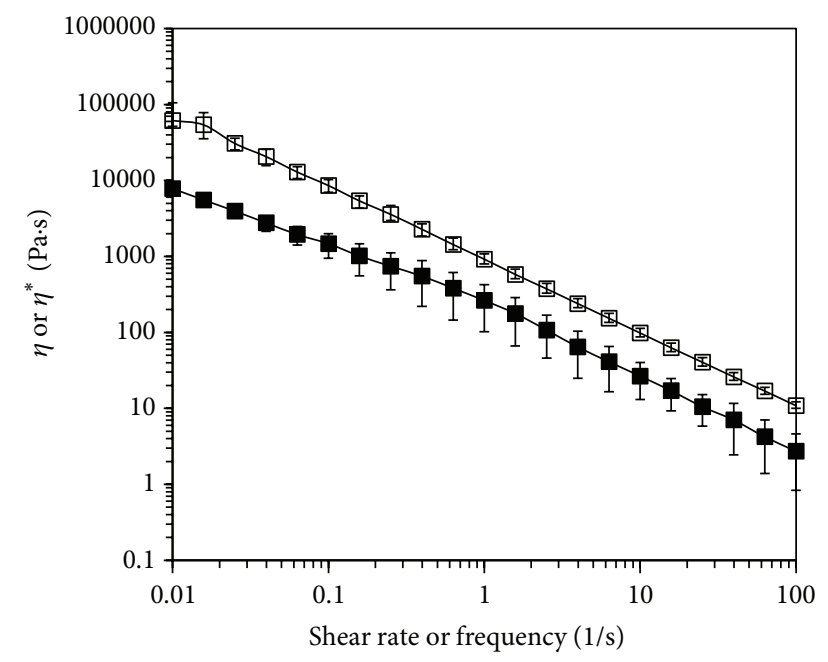

FIgURE 6: Example of the Cox-Merz rule not being obeyed: 1.6 vol.\% Ag-EG. Constant shear viscosity $\eta$ (solid squares) and complex viscosity $\eta^{*}$ (open squares).

in a vacuum oven for over 2 hours at $80^{\circ} \mathrm{C}$ under $-30 \mathrm{mmHg}$ vacuum. A worm texture, named for the constant fluctuation of domains under flow, resulted. Similar textures have been previously observed in liquid crystals $[38,39]$. Figure 7 shows that the densely packed disclinations are generally aligned along the shearing direction. The dispersions' polydomain structure remained, due to the high disclination density associated with the relatively high shear rate [21] and the demixing of silver nanowires and nanoparticle aggregates. This worm texture could be preserved after cessation of shear and even after solvent removal. We believe that the high stability of the worm-like structure is caused by the large nanowire persistence lengths and attractive interactions as well as the demixed nematic phase formation reported in our previous paper [18]. These considerations also explain why the worm-like texture was obtained at much higher shear rates than 1 to $100 \mathrm{~s}^{-1}$ [37] observed for liquid crystalline polymers $[39,40]$.

In addition to worm textures, shear band textures were also achieved. Shear band textures which were first observed in liquid crystalline dispersions used to make Kevlar fibers [41] are interesting structures that can form during shear or after the cessation of shear. They are commonly found in both thermotropic and lyotropic liquid crystal systems after they are processed by thin film shearing [42, 43], uniaxial fiber drawing [44], and injection molding [45]. Dark bands lie perpendicular to the direction of shear and represent a periodic fluctuation of molecules aligned around the shear direction. This periodic oscillation of molecules ordering director is induced by the strain relaxation (stored elastic energy of the texture) after cessation of shear $[39,46]$ or during continuous shear $[43,47]$.

As shown in Figure 8, thin films with $1-2 \mu \mathrm{m}$ thickness made by unidirectional shearing of a biphasic dispersion of 0.8 vol. $\% ~ A g-\mathrm{H}_{2} \mathrm{O}$ at $500 \mathrm{~s}^{-1}$ resulted in a banded texture. The tilt angle of molecules relative to the shearing direction was determined using the method introduced by Wang and Labes [48]. In this method, the polarizer and analyzer are kept crossed, the bands are initially positioned perpendicular to either the polarizer or analyzer, and then the microscope stage is rotated to make the image change as a function of rotation angle. When the molecules in the bands are aligned with either polarizer or analyzer, the bands will appear completely dark. The tilt angle can then be calculated by the rotated angle of the stage. In this case, when the polarizer and analyzer are crossed and the direction of shear is parallel to the polarizer direction, the polarized light microscopy image in Figure 8(a) exhibited bright bands and dark lines which alternated and were perpendicular with the shear direction (stage angle equal to $0^{\circ}$ ). When the microscopy sample stage was rotated counterclockwise with an angle $\theta=53^{\circ}$ the bright bands turned completely dark and the remaining bands were bright as shown in Figure 8 (b). When the stage was rotated clockwise with an angle of $\theta=53^{\circ}$, the dark bands shown in Figure 8(b) turned bright and the bright bands in Figure 8(b) turned dark as shown in Figure 8(c). In this case, the tilt angle $\alpha$ of molecules relative to the direction of shear was $\alpha=90^{\circ}-53^{\circ}=37^{\circ}$. For polymer liquid crystals, the band size was found to be a function of shear rate, polymer molecular weight, sample thickness, solution concentration, type of solvent and solvent evaporation rate, and other experimental variables [49]. In this study, silver nanowire thin films made by shearing $300 \mu \mathrm{m}$ thick 0.8 vol. $\%$ Ag- $\mathrm{H}_{2} \mathrm{O}$ biphasic dispersions at $500 \mathrm{~s}^{-1}$ shear rate showed band sizes from 10 to $70 \mu \mathrm{m}$ in width. This band size range is significantly larger than the few micrometers which was reported for a model system, aqueous hydroxypropyl cellulose (HPC) [46]. This may be due to the significant larger persistence length of silver nanowires. It should be noted that the $\mathrm{Ag}-\mathrm{H}_{2} \mathrm{O}$ banded texture was only observed at shear rates higher than $500 \mathrm{~s}^{-1}$. This is consistent with previous observations on HPC, which showed that the banded texture can only form when the magnitude of shear rate exceeded a certain threshold value $[46,50,51]$.

The ability to control microstructure in dried coatings/ films suggests the ability to create structures with anisotropic properties. For example, the electrical resistivity of a silver nanowire thin film with shear banding texture was characterized in both the parallel and perpendicular direction relative to the silver nanowire alignment. The sheet resistance of the silver thin film was measured using the four-point probe method. The geometric correction factor $F$, which is usually required for sheet resistance calculations by the fourpoint probe method, was simplified to $F=(t / s) / \ln (2), t$ is the thickness of the thin film, and $s$ is the probe spacing. Based on the measured voltage/current ratio $V / I$, the sheet resistances for thin film were calculated by the equation $R_{\mathrm{sh}}=$ $2 \pi(s / t) F(V / I)$, so $R_{\text {sh }}=(\pi / \ln (2))(V / I)$. The extracted sheet resistance and corresponding resistivity of silver film in the direction parallel to the alignment were $R_{\mathrm{sh} \|}=0.71 \Omega / \mathrm{sq}$ and $\rho_{\|}=95 \mu \Omega \cdot \mathrm{cm}$, while the sheet resistance in the direction perpendicular to the alignment was $R_{\mathrm{sh} \perp}=1.3 \Omega / \mathrm{sq}, \rho_{\perp}=$ $221 \mu \Omega \cdot \mathrm{cm}$. The ratio of resistivity perpendicular/parallel to the alignment direction is about 2.3 times. The sheet resistance and resistivity in both directions are much smaller 


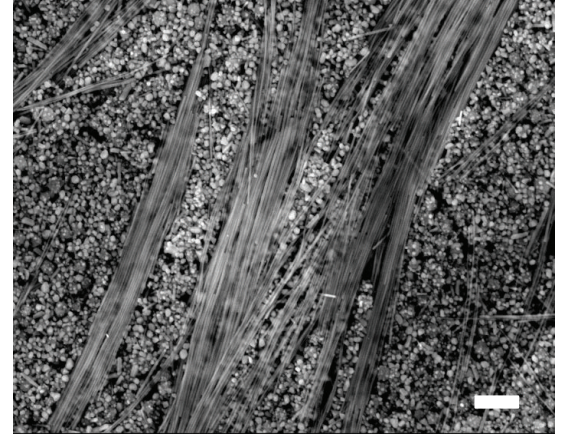

(a)

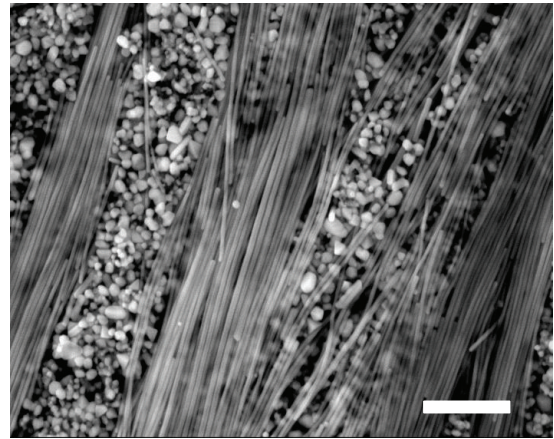

(b)

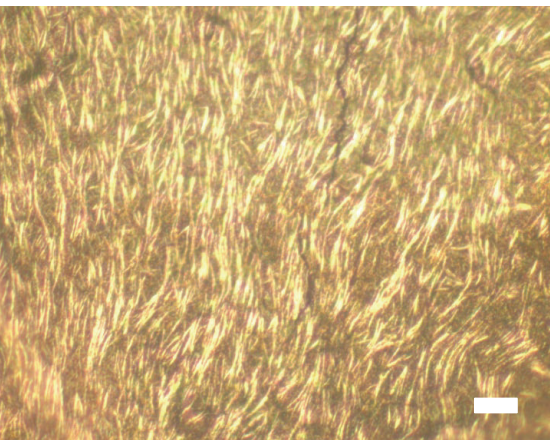

(c)

Figure 7: $(\mathrm{a}, \mathrm{b})$ Scanning electron microscopy images of a dried coating made by shearing a $1.1 \mathrm{vol} . \%$ Ag- $\mathrm{H}_{2} \mathrm{O}$ dispersion at $200 \mathrm{~s}^{-1}$; the images show a worm-like texture with all the liquid crystalline domains generally aligned in the shearing direction. (c) Optical image of the same coating under reflective cross-polarized light using a 20x 0.45 NA objective with $2 \mathrm{x}$ additional magnification in front of the camera. Scale bars are $1 \mu \mathrm{m}$ in (a) and (b) and $20 \mu \mathrm{m}$ in (c).
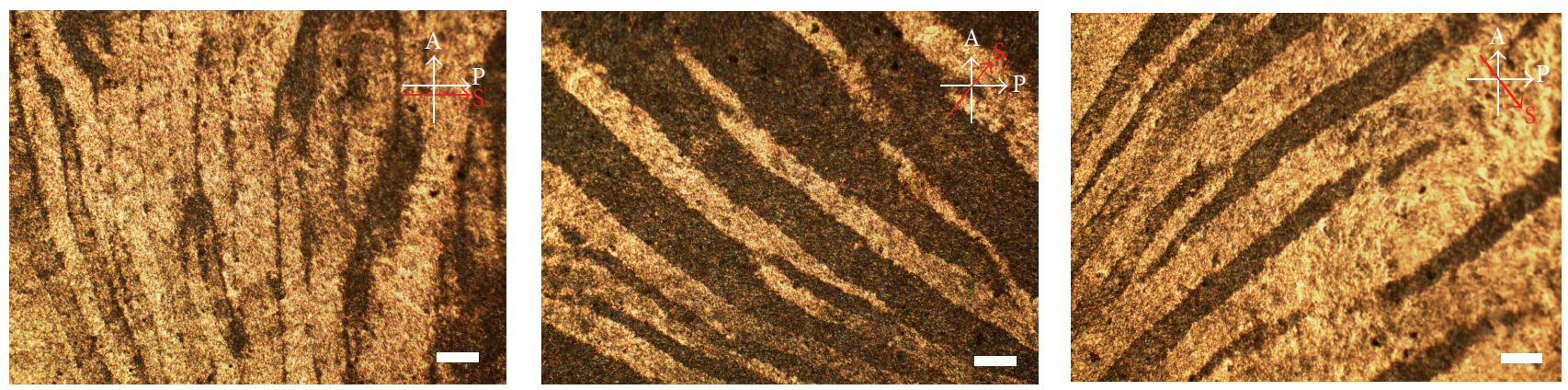

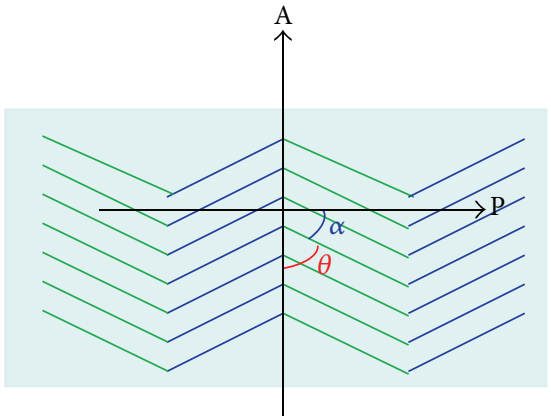

(a)

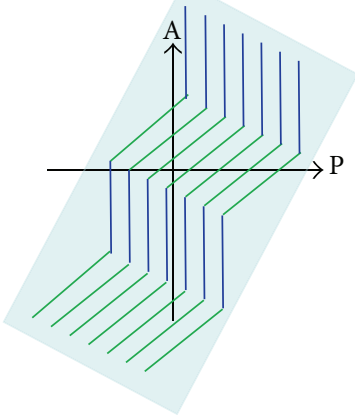

(b)

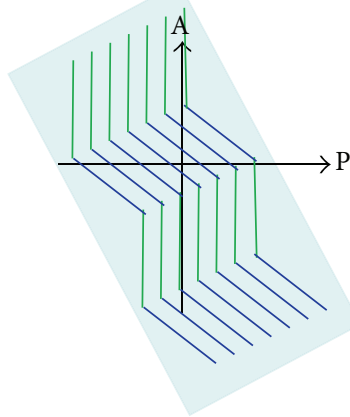

(c)

FIGURE 8: Cross-polarized optical microscopy showing the banded texture of silver nanowire thin films made by unidirectional shear at $500 \mathrm{~s}^{-1}$ followed by drying in a vacuum oven. (a) Direction of shear parallel to the polarizer; (b) stage was rotated clockwise with $53^{\circ}$; (c) stage was rotated counterclockwise with $53^{\circ}$. All scale bars represent $40 \mu \mathrm{m}$. A: analyzer; P: polarizer; S: the direction of shear.

than a previous report on silver nanoparticle thin films [52] but highlight the potential for anisotropy. The relatively high resistance is attributed to defects in the dried films and the presence of residual PVP on the surface of the silver nanomaterials.

\section{Conclusion}

Dispersions of high aspect ratio silver nanowires and large nanoparticle aggregates dispersions can form nematic liquid crystalline phases. The rheological behaviors of the dispersion are similar to those typically associated with lyotropic liquid crystalline polymers (LCPs). This was somewhat surprising given the system's complexity and the relatively low number fraction of rods. The results highlight the significant effect the rotational diffusivity of high aspect ratio rods has on rheological behavior compared to low aspect ratio particles. Shearing predominantly liquid crystalline dispersions resulted in shear rate dependent structures with both alignment and demixing. A worm-like texture of silver nanowires was formed by applying a $200 \mathrm{~s}^{-1}$ shear rate to anisotropic dispersions. A banded texture of silver nanowires formed at $500 \mathrm{~s}^{-1}$ shear rate with uniform alignment at the top of the coating and nanospheres being segregated to the bottom of 
the coating. The ability to control nanowire alignment and segregate rods and spheres opens up intriguing possibilities for the single-step manufacture of coatings with complex architectures and compositions simply by controlling phase behavior and applied shear.

\section{Conflict of Interests}

The authors declare that there is no conflict of interests regarding the publication of this paper.

\section{Acknowledgments}

The authors would like to acknowledge Dr. Michael Hamilton and Pingye $\mathrm{Xu}$ for silver nanowire thin film electric property characterization. This research was supported by a National Science Foundation CAREER Grant (CMMI-0846629).

\section{References}

[1] M. R. Jones, K. D. Osberg, R. J. MacFarlane, M. R. Langille, and C. A. Mirkin, "Templated techniques for the synthesis and assembly of plasmonic nanostructures," Chemical Reviews, vol. 111, no. 6, pp. 3736-3827, 2011.

[2] X. Zhou, Y. Zhou, J. C. Ku, C. Zhang, and C. A. Mirkin, "Capillary force-driven, large-area alignment of multi-segmented nanowires," ACS Nano, vol. 8, no. 2, pp. 1511-1516, 2014.

[3] K. M. M. Abou El-Nour, A. Eftaiha, A. Al-Warthan, and R. A. A. Ammar, "Synthesis and applications of silver nanoparticles," Arabian Journal of Chemistry, vol. 3, no. 3, pp. 135-140, 2010.

[4] S.-W. Lee, S. K. Lee, and A. M. Belcher, "Virus-based alignment of inorganic, organic, and biological nanosized materials," Advanced Materials, vol. 15, no. 9, pp. 689-692, 2003.

[5] H. Ko and V. V. Tsukruk, "Liquid-crystalline processing of highly oriented carbon nanotube arrays for thin-film transistors," Nano Letters, vol. 6, no. 7, pp. 1443-1448, 2006.

[6] X. F. Duan, "Assembled semiconductor nanowire thin films for high-performance flexible macroelectronics," MRS Bulletin, vol. 32, no. 2, pp. 134-141, 2007.

[7] X. Gong, "Facile formation of nanoparticle patterns by water induced flow of a polymer thin film," RSC Advances, vol. 4, pp. 54494-54499, 2014.

[8] Q. Xu, M. Li, L. Zhang, J. Niu, and Z. Xia, "Dynamic adhesion forces between microparticles and substrates in water," Langmuir, vol. 30, no. 37, pp. 11103-11109, 2014.

[9] J.-Q. Xi, J. K. Kim, and E. F. Schubert, "Silica nanorod-array films with very low refractive indices," Nano Letters, vol. 5, no. 7, pp. 1385-1387, 2005.

[10] M. H. Huang, S. Mao, H. Feick et al., "Room-temperature ultraviolet nanowire nanolasers," Science, vol. 292, no. 5523, pp. 18971899, 2001.

[11] X. F. Duan, C. M. Niu, V. Sahi et al., "High-performance thinfilm transistors using semiconductor nanowires and nanoribbons," Nature, vol. 425, pp. 274-278, 2003.

[12] W. U. Huynh, J. J. Dittmer, and A. P. Alivisatos, "Hybrid nanorod-polymer solar cells," Science, vol. 295, no. 5564, pp. 24252427, 2002.

[13] A. Saha, C. Jiang, and A. A. Martí, "Carbon nanotube networks on different platforms," Carbon, vol. 79, no. 1, pp. 1-18, 2014.
[14] S. B. Chaney, S. Shanmukh, R. A. Dluhy, and Y.-P. Zhao, "Aligned silver nanorod arrays produce high sensitivity surfaceenhanced Raman spectroscopy substrates," Applied Physics Letters, vol. 87, no. 3, Article ID 031908, 2005.

[15] Y. Yang, L. Xiong, J. Shi, and M. Nogami, "Aligned silver nanorod arrays for surface-enhanced Raman scattering," Nanotechnology, vol. 17, no. 10, pp. 2670-2674, 2006.

[16] Z.-A. Hu, Y.-X. Wang, Y.-L. Xie, Y.-Y. Yang, Z.-Y. Zhang, and H.$\mathrm{Y}$. Wu, "Ag nanowires and its application as electrode materials in electrochemical capacitor," Journal of Applied Electrochemistry, vol. 40, no. 2, pp. 341-344, 2010.

[17] S. De, T. M. Higgins, P. E. Lyons et al., "Silver nanowire networks as flexible, transparent, conducting films: extremely high DC to optical conductivity ratios," ACS Nano, vol. 3, no. 7, pp. 1767$1774,2009$.

[18] S. Murali, T. Xu, B. D. Marshall et al., "Lyotropic liquid crystalline self-assembly in dispersions of silver nanowires and nanoparticles," Langmuir, vol. 26, no. 13, pp. 11176-11183, 2010.

[19] G. Kiss and R. S. Porter, "Rheology of concentrated solutions of poly( $\gamma$-benzyl-glutamate)," Journal of Polymer Science: Polymer Symposia, vol. 65, no. 1, pp. 193-211, 1978.

[20] G. Kiss and R. S. Porter, "Rheology of concentrated solutions of poly( $\gamma$-benzyl-glutamate)," Journal of Polymer Science, Part B: Polymer Physics, vol. 34, no. 14, pp. 2271-2289, 1996.

[21] R. G. Larson, The Structure and Rheology of Complex Fluids, Oxford University Press, New York, NY, USA, 1999.

[22] A. Ciferri, Rheology of Nematic Polymers. Liquid Crystallinity in Polymers, VCH Publishers, New York, NY, USA, 1991.

[23] L. F. Gou, M. Chipara, and J. M. Zaleski, "Convenient, rapid synthesis of Ag nanowires," Chemistry of Materials, vol. 19, no. 7, pp. 1755-1760, 2007.

[24] V. A. Davis, L. M. Ericson, A. N. G. Parra-Vasquez et al., "Phase behavior and rheology of SWNTs in superacids," Macromolecules, vol. 37, no. 1, pp. 154-160, 2004.

[25] M. Adams, Z. Dogic, S. L. Keller, and S. Fraden, "Entropically driven microphase transitions in mixtures of colloidal rods and spheres," Nature, vol. 393, no. 6683, pp. 349-352, 1998.

[26] G. Ao, D. Nepal, M. Aono, and V. A. Davis, "Cholesteric and nematic liquid crystalline phase behavior of double-stranded DNA stabilized single-walled carbon nanotube dispersions," ACS Nano, vol. 5, no. 2, pp. 1450-1458, 2011.

[27] V. A. Davis, "Liquid crystalline assembly of nanocylinders," Journal of Materials Research, vol. 26, no. 2, pp. 140-153, 2011.

[28] V. A. Davis, A. N. G. Parra-Vasquez, M. J. Green et al., "True solutions of single-walled carbon nanotubes for assembly into macroscopic materials," Nature Nanotechnology, vol. 4, no. 12, pp. 830-834, 2009.

[29] E. E. Ureña-Benavides, G. Ao, V. A. Davis, and C. L. Kitchens, "Rheology and phase behavior of lyotropic cellulose nanocrystal suspensions," Macromolecules, vol. 44, no. 22, pp. 8990-8998, 2011.

[30] M. J. Solomon and P. T. Spicer, "Microstructural regimes of colloidal rod suspensions, gels, and glasses," Soft Matter, vol. 6, no. 7, pp. 1391-1400, 2010.

[31] T. Xu and V. A. Davis, "Liquid crystalline phase behavior of silica nanorods in dimethyl sulfoxide and water," Langmuir, vol. 30, no. 16, pp. 4806-4813, 2014.

[32] G. Marrucci, "Rheology of liquid crystalline polymers," Physica Scripta, vol. 1991, no. 35, pp. 44-46, 1991.

[33] L. M. Walker, W. A. Kernick III, and N. J. Wagner, "In situ analysis of the defect texture in liquid crystal polymer solutions under shear," Macromolecules, vol. 30, no. 3, pp. 508-514, 1997. 
[34] R. G. Larson and D. W. Mead, "The Ericksen number and Deborah number cascades in sheared polymeric nematics," Liquid Crystals, vol. 15, no. 2, pp. 151-169, 1993.

[35] W.-M. Kulicke, H.-E. Jeberien, H. Kiss, and R. S. Porter, "Visual observation of flow irregularities in polymer solutions at thetaconditions," Rheologica Acta, vol. 18, no. 6, pp. 711-716, 1979.

[36] M. J. Green, "Isotropic-nematic phase separation and demixing in mixtures of spherical nanoparticles with length-polydisperse nanorods," Journal of Polymer Science, Part B: Polymer Physics, vol. 50, no. 18, pp. 1321-1327, 2012.

[37] A. Donald, A. Windle, and S. Hanna, Liquid Crystalline Polymers, Cambridge University Press, Cambridge, UK, 2nd edition, 2006.

[38] T. De'Nève, P. Navard, and M. Kléman, "Shear rheology and shear-induced textures of a thermotropic copolyesteramide," Journal of Rheology, vol. 37, pp. 515-529, 1993.

[39] N. J. Alderman and M. R. Mackley, "Optical textures observed during the shearing of thermotropic liquid-crystal polymers," Faraday Discussions, vol. 79, pp. 149-160, 1985.

[40] P. T. Mather, D. S. Pearson, and R. G. Larson, "Flow patterns and disclination-density measurements in sheared nematic liquid crystals II: tumbling 8CB," Liquid Crystals, vol. 20, no. 5, pp. 539-546, 1996

[41] M. G. Dobb, D. J. Johnson, and B. P. Saville, "Supramolecular structure of a high-modulus polyaromatic fiber (Kevlar 49)," Journal of Polymer Science Part B: Polymer Physics, vol. 15, no. 12, pp. 2201-2211, 1977.

[42] A. M. Donald, C. Viney, and A. H. Windle, "Banded structures in oriented thermotropic polymers," Polymer, vol. 24, no. 2, pp. 155-159, 1983.

[43] G. Kiss and R. S. Porter, "Rheo-optical studies of liquid crystalline solutions of helical polypeptides," Molecular Crystals and Liquid Crystals, vol. 60, no. 4, pp. 267-280, 1980.

[44] S. C. Simmens and J. W. S. Hearle, "Observation of bands in high-modulus aramid fibers by optical microscopy," Journal of Polymer Science Part B-Polymer Physics, vol. 18, no. 4, pp. 871876, 1980.

[45] H. Thapar and M. Bevis, "The micromorphology of an injection moulded thermotropic liquid crystal polymer," Journal of Materials Science Letters, vol. 2, no. 12, pp. 733-736, 1983.

[46] B. Ernst and P. Navard, "Band textures in mesomorphic (hydroxypropyl) cellulose solutions," Macromolecules, vol. 22, no. 3, pp. 1419-1422, 1989.

[47] P. Navard, "Formation of band textures in hydroxypropylcellulose liquid crystals," Journal of Polymer Science Part B: Polymer Physics, vol. 24, no. 2, pp. 435-442, 1986.

[48] J. Wang and M. M. Labes, "Control of the anisotropic mechanical properties of liquid crystal polymer films by variations in their banded texture," Macromolecules, vol. 25, no. 21, pp. 57905793, 1992.

[49] C. Viney and W. S. Putnam, "The banded microstructure of sheared liquid-crystalline polymers," Polymer, vol. 36, no. 9, pp. 1731-1741, 1995.

[50] G. Marrucci, N. Grizzuti, and A. Buonaurio, "Band formation in sheared HPC solutions. effects of sample thickness," Molecular Crystals and Liquid Crystals, vol. 153, no. 1, pp. 263-269, 1987.

[51] E. Marsano, L. Carpaneto, and A. Ciferri, "Formation of a banded texture in solutions of liquid crystalline polymers: 1 . Hydroxypropylcellulose in $\mathrm{H}_{2} \mathrm{O}$," Molecular Crystals and Liquid Crystals Incorporating Nonlinear Optics, vol. 158, no. 2, pp. 267$278,1988$.
[52] M. S. Miller, J. C. O’Kane, A. Niec, R. S. Carmichael, and T. B. Carmichael, "Silver nanowire/optical adhesive coatings as transparent electrodes for flexible electronics," ACS Applied Materials and Interfaces, vol. 5, no. 20, pp. 10165-10172, 2013. 

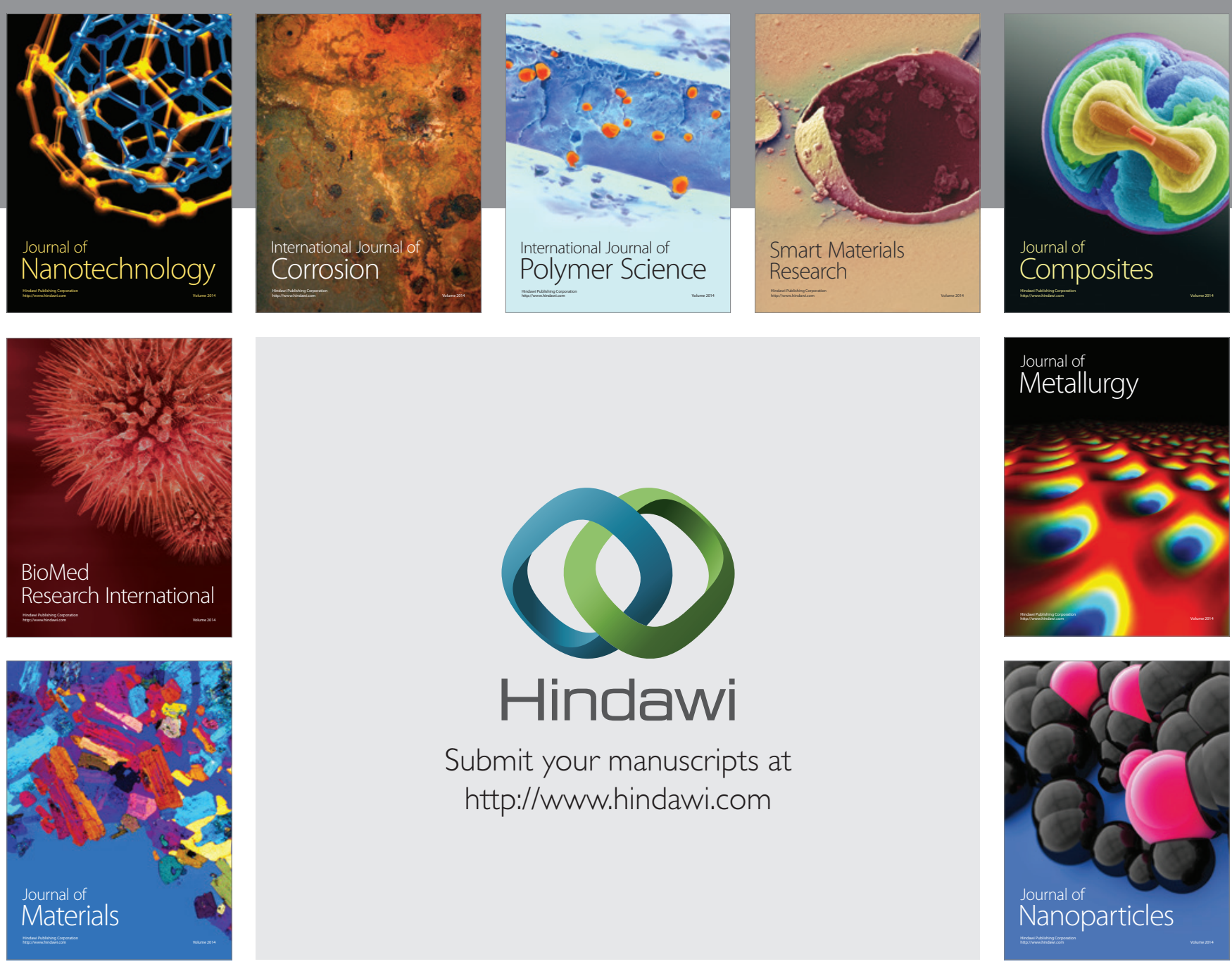

Submit your manuscripts at http://www.hindawi.com
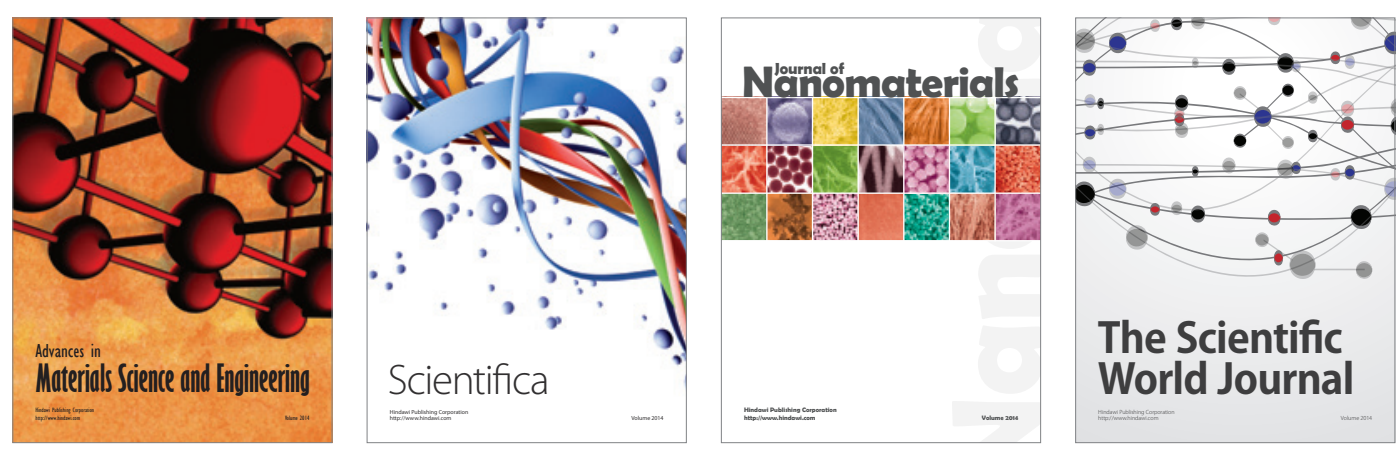

\section{The Scientific World Journal}
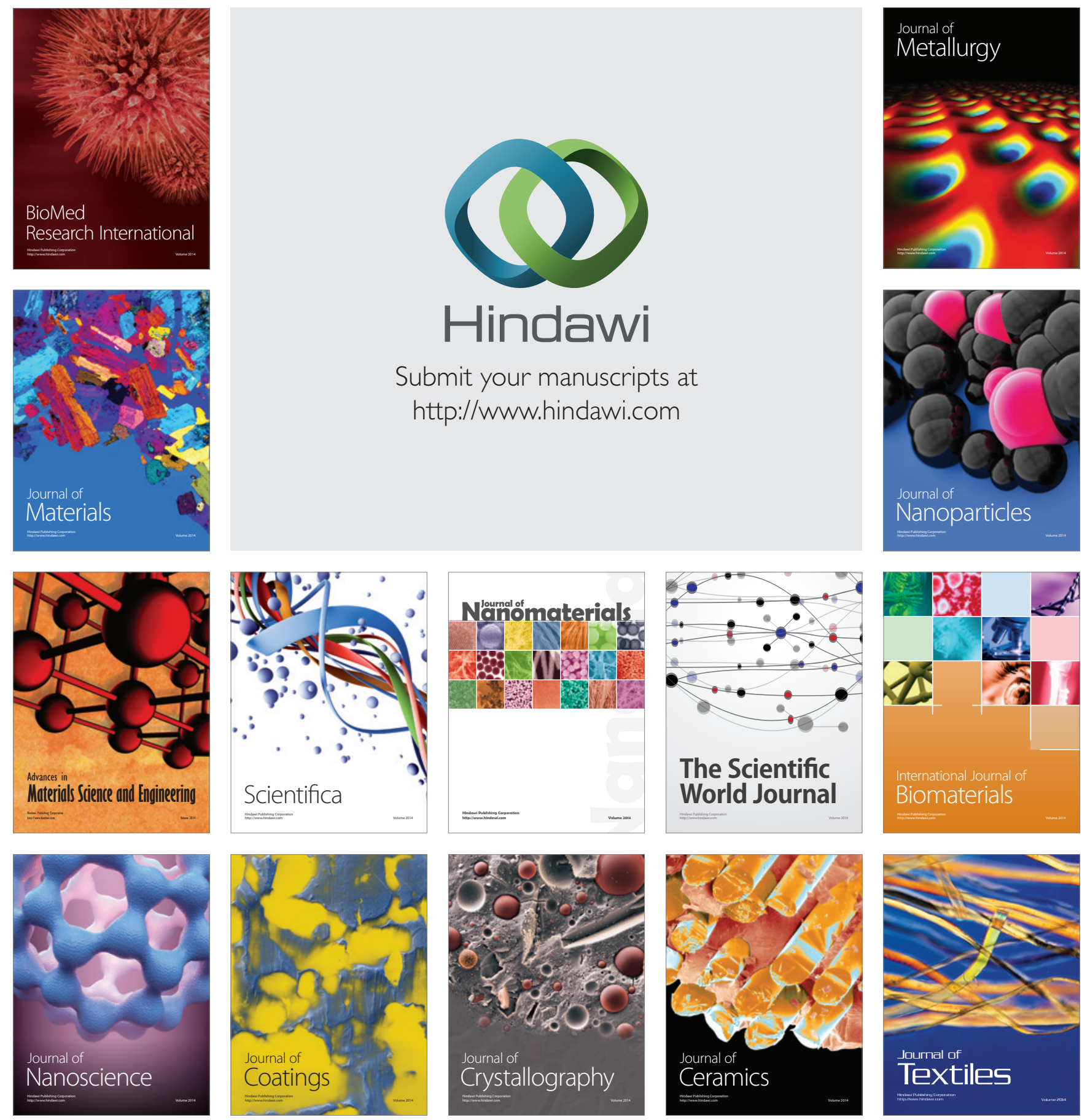